\author{
Revista Calidad en la Educación Superior \\ Programa de Autoevaluación Académica \\ Universidad Estatal a Distancia, Costa Rica \\ ISSN 1659-4703 \\ revistacalidad@uned.ac.cr
}

\title{
EL DERECHO A LA EDUCACIÓN EN COSTA RICA: ENTRE LA REALIDAD Y LA UTOPIA
}

\author{
THE RIGHT TO EDUCATION IN COSTA RICA: BETWEEN REALITY AND \\ UTOPIA
}

\author{
Carlos Alberto Rodríguez Ramírez ${ }^{1}$ \\ caberoro@gmail.com \\ Escuela de Filosofía, Universidad de Costa Rica
}

"Básicamente, siempre educamos para un mundo que está confuso o se está convirtiendo en confuso, porque ésta es la situación humana básica en la que se creó el mundo por acción de manos mortales para servir a los mortales como hogar durante un tiempo limitado. Porque está hecho por mortales, el mundo se marchita; y porque continuamente cambian sus habitantes, corre el riesgo de llegar a ser tan mortal como ellos. Para preservar al mundo del carácter mortal de sus creadores y habitantes hay que volver a ponerlo, una y otra vez, en el punto justo" (Hannah Arendt, p.204)

\author{
Volumen 6, Número 1 \\ Mayo 2015 \\ Pp. $249-268$
}

Recibido: 12 de marzo, 2015

Aprobado: 10 de abril, 2015

\footnotetext{
${ }^{1}$ Carlos Alberto Rodríguez Ramírez, Profesor Asociado de Filosofía, Escuela de Filosofía Universidad de Costa Rica.
} 


\section{Resumen}

Con base en el concepto de educación adoptado por la UNESCO y la ONU, el artículo se enfoca en el derecho a la educación en el ámbito costarricense, con el propósito de realizar un balance crítico sobre su estado actual.

Palabras claves: Educación; política; Estado; presupuesto; niñez; adolescencia.

\section{Abstract}

Based on the concept of education adopted by UNESCO and the UN, this article focuses on the right to education in the Costa Rican context, in order to make a critical assessment of the current status.

Keywords: Education; political; state; budget; childhood; adolescence

El 24 octubre de1945, en medio de un convulso siglo XX, y con el antecedente negativo de dos Guerras Mundiales, se funda la Organización de las Naciones Unidas (ONU). Es comprensible que en medio de criterios ideológicos y visiones de mundo tan dispares, los consensos fueran (y son) difíciles, más no imposibles. Precisamente, en el marco de esta organización emerge la Declaración Universal de los Derechos Humanos, el 10 de diciembre de 1948, que desde entonces hasta la actualidad ha venido a ser una característica central de convivencia y socialización en el mundo contemporáneo. En efecto, los derechos humanos se han convertido en un tema del que la humanidad no puede ser indiferente, aunque estos derechos no siempre sean respetados, lo cierto es que a partir de esta Declaración de la ONU, se generarán cambios en las jurisprudencias internas de los Estados, aunque no necesariamente sean vinculantes jurídicamente, pero sí moralmente, incentivará el desarrollo de nuevas Declaraciones y la sociedad civil tomará un papel más activo en pro de la defensa de los derechos humanos. 
Esta Declaración es muy importante, entre otras razones porque tiene una pretensión universal y marcará el tema de los derechos humanos inevitablemente. En este sentido, en este trabajo se hace referencia a la misma por dos razones: la primera se debe a que alude al derecho a la educación (artículo 26) con carácter gratuito y obligatorio -al menos en lo concerniente a la instrucción elemental- para permitir "el pleno desarrollo de la personalidad humana y el fortalecimiento del respeto a los derechos humanos y las libertades fundamentales". La segunda es que siguiendo esta línea se promoverán posteriormente, otras declaraciones y acuerdos, tales como la Declaración de los Derechos del Niño, de la Mujer, de las personas migrantes o en situaciones especiales, entre otros, donde el tema de la educación está presente.

Precisamente, el tema de interés de este artículo se centra en el derecho a la educación en el ámbito costarricense, y pretende analizar a partir de la revisión bibliográfica de algunos documentos, las pretensiones, propuestas y realidades de la educación formal de nuestro país. Para tal efecto, se considera el Cuarto Informe del Estado de la Educación del 2013, se revisará este derecho a la educación en los niveles de preescolar, básica y secundaria, y se realizan reflexiones propias al respecto. Lo que subyace en el fondo, es que contrario a lo que defienden algunos sectores económicos y políticos locales respecto a que hay una mayor cobertura de educación formal escolarizada, se pretende evidenciar que la brecha social se está ensanchando, con lo cual se violenta este derecho a la educación.

\section{¿Qué es derecho a la educación?}

Una de las 12 tesis que propone Rosa María Torres (2005) para un cambio educativo afirma que el derecho a la educación es más que el derecho a matricular en un sistema escolar "El objetivo de la educación -y por tanto el 
derecho a la educación- es aprender, aprender a aprender y aprender a poner en práctica conocimientos, habilidades, valores y actitudes útiles para la vida y para continuar aprendiendo" (p.74). Señala también que la palabra "derecho" se volvió a activar a partir de la Conferencia Mundial de Educación 2000, celebrada en Dakar, donde se le asocia con las necesidades básicas de aprendizaje, y que se correlacionan con los derechos económicos, sociales y culturales, que no siempre se respetan.

Jacques Delors (1999, p.7) habla de la educación como una utopía necesaria, y «como un instrumento indispensable para que la humanidad pueda progresar hacia los ideales de paz, libertad y justicia social». Esto supone que es un área humana vital, que no está acabada, sino que constantemente evoluciona y que es el medio idóneo para alcanzar sociedades justas y equitativas. Supone también, que tiene dos contextos, uno lo que idealiza y busca alcanzar la "utopía necesaria" que debe ser el ingrediente básico presente en cualquier objetivo educativo; el otro, el desarrollo de la labor educativa en comunidades y naciones, que tienen grandes limitaciones económicas, académicas, infraestructurales, entre otras.

En plena Segunda guerra mundial (1942) hubo una reunión de ministros de educación de los países aliados, buscando en la educación un medio real para regenerar la paz. No fue sino hasta 1945, cuando el conflicto bélico finalizó, que esta idea dio paso la creación de la UNESCO y se afirmó que "Dentro de su espíritu, esta nueva organización debe establecer la "solidaridad intelectual y moral de la humanidad" y, de esta manera, impedir que se desencadene una nueva guerra mundial" (UNESCO, 2011, párr.1) Este organismo considera que como parte del derecho a la educación está el reconocer la obligatoriedad, gratuidad y universalidad de la educación primaria, porque es una etapa fundamental para eliminar las desigualdades, discriminaciones y mejorar la calidad de vida de los seres humanos. 
Ese espíritu está presente en el artículo 26 de la Declaración Universal de los Derechos Humanos, que estipula como un derecho humano fundamental el de la educación. De manera específica aparecerá de nuevo de este derecho en la Declaración de los Derechos del Niño (1959), en la Convención sobre los Derechos del Niño (1989) y en Pacto Internacional de Derechos Económicos, Sociales y Culturales. Además, posterior a dicho suceso, la Comisión de Derechos Humanos de la ONU crea las Relatorías Especiales, entre las que está la del derecho a la educación, cuyo propósito es investigar la situación de la educación en los países miembros, realizar denuncias cuando lo amerite y realizar informes temáticos a la Comisión y a la Asamblea General. Dentro de este contexto, los diferentes informes que han ido surgiendo han focalizado problemáticas de grupos que no tienen acceso y están excluidos por diversas causas del derecho a la educación.

Cuando se hace referencia al derecho a la educación es inevitable considerar que deba canalizarse a través de una normativa jurídica, que no es suficiente, pero que es necesaria, por lo que la UNESCO afirma que este derecho "consiste en velar porque las obligaciones contraídas por los Estados Miembros se incorporen a los sistemas jurídicos nacionales» (UNESCO, 2011, párr.1). Al respecto es pertinente lo que afirma Méndez (2014, p.11) en su texto en cuanto que se "pretende posicionar la discusión sobre los alcances, fundamentos y coincidencias entre la retórica legal y la vivencia de derechos humanos, tomando en consideración la funcionalidad económica que sustente muchos de los planteamientos relativos a la niñez y adolescencia". En otras palabras, que aunque aparezca este derecho a la educación en una normativa jurídica, no es garantía de que corresponda con una protección integral. De ahí el importante papel de vigilancia para que se respete este derecho, a través de instancias como las Relatorías Especiales de la ONU, y las organizaciones de la sociedad civil. 
Cuando Vernor Muñoz se refiere a la educación como parte esencial de la cultura, la relaciona con los procesos de aprendizaje que deben desarrollarse como parte esencial de la vida. A partir de esta visión amplia e inclusiva, considera "que la educación tiene una legitimidad ética, filosófica y científica indubitable -una ontología propia-, y es por ello que el derecho a la educación no sólo constituye una garantía para la humanidad, sino un fundamento para la ecología y la igualdad social" (Muñoz, 2009, p.17).

La educación, por lo tanto, es un derecho fundamental, pero debe correlacionarse con los otros derechos humanos para que se pueda implementar apropiadamente, porque de lo contrario, quedaría en el plano meramente utópico, y eso es lo que sucede sino se consideran las áreas económicas, políticas y culturales que predominan en las Naciones. De esta forma, el Informe Delors se afirma en el capítulo 3:

"El establecimiento de nuevas relaciones entre política de educación y política de desarrollo con objeto de fortalecer las bases del saber teórico y técnico en los países interesados: incitación a la iniciativa, al trabajo en equipo, a las sinergias realistas en función de los recursos locales, al autoempleo y al espíritu de empresa" (Delors, 1999, p.33).

En el caso específico de Costa Rica, como país miembro de estas instancias organizacionales mencionadas, asume los acuerdos y declaraciones que emiten. Además, las contempla en la jurisprudencia interna de la Nación. Así, el derecho a la educación aparece en la Constitución Política, el Título VII, artículos del 76 al 89, que dedica a la educación y la cultura. Es relevante, destacar el artículo 77 que establece una organización de la educación pública que correlacione lo preescolar hasta lo universitario; el artículo 78, que enfatiza que la educación general, desde preescolar hasta la diversificada, debe ser gratuita y obligatoria, así como la obligación del Estado de proveer apoyo financiero a estudiantes de 
educación superior que carezcan de estos recursos; y el artículo 83 que menciona la educación de adultos para combatir el analfabetismo y la apertura de oportunidades a quienes no las tengan.

Otro documento importante que garantiza el derecho a la educación en nuestro país es la Ley Fundamental de Educación, cuyos fines establecen en el artículo 1 que "Todo habitante de la República tiene derecho a la educación y el Estado tiene la obligación de procurar ofrecerla en la forma más amplia y adecuada". Asimismo, el artículo 6 cuando se refiere al Sistema Educativo Nacional, menciona la educación escolar y extraescolar; la primera se organiza de acuerdo con lo que dice el siguiente artículo (art. 7) en 4 ciclos: educación preescolar, primaria, media y superior.

Un par de detalles antes de continuar con el análisis. Torres afirma que toda política específica, la que incluye la educativa, debe verse necesariamente en un marco más amplio de la política en su conjunto, porque "la política económica, la política fiscal, la política social, la política exterior, la política de cooperación internacional, inciden en la educación tanto o más que aquello que acostumbramos llamar "presupuesto educativo" y "política educativa"” (2005, p.12). En este sentido, cabe señalar que la educación es el reflejo de una sociedad y de su situación política. También esta autora hace otra valiosa aportación que se avala en este texto: educación no puede reducirse a educación formal escolar, pues la que se asocia con un sistema educativo, es mucho más amplia, abarca también la educación no formal, (familia, trabajo, espacios de comunicación e información, entre otros). En otras palabras, el sistema escolar no es el único sistema de aprendizaje, por lo que es injusto atribuirle toda la responsabilidad a este sistema y esperar las soluciones a todas las necesidades de aprendizaje, o reducir este derecho solo a la niñez, porque "tanto derecho a la educación tiene un niño o niña, como un joven o una persona adulta. Aprender a 
leer y escribir comprensivamente es un derecho fundamental de toda persona, dentro o fuera del sistema escolar, a cualquier edad" (Torres, 2005, p.63).

\section{La "gratuidad y obligatoriedad" en la educación preescolar}

Desde hace 20 años se realizan estudios sobre la evolución del estado de la Nación en sus diversos campos, lo que incluye por supuesto la educación, de la que, de manera específica, se han emitido cuatro informes. La característica de los Informes que se publican es que tienen un carácter independiente, sus resultados no son vinculantes, aunque tienen un gran impacto en la opinión pública, y de una u otra manera, influyen en las políticas públicas que se desarrollen. En este y el siguiente apartado se harán referencias al Cuarto Informe del Estado de la Nación sobre la educación, que es del 2013 y el que está vigente. El temario del quinto informe sobre el estado de la educación se aprobó en marzo del 2014, con el propósito de actualizar la información y evidenciar nuevos datos.

En el Cuarto Informe, capítulo 2 en la sección de "hallazgos relevantes" dice que "uno de cada tres niños de entre 0 a 6 años viven en un hogar pobre. El $41.2 \%$ de los niños menores de 7 años reside en hogares cuyos miembros tienen bajan escolaridad" (Cuarto informe Estado de la Nación, 2013, p.77), lo que incide también en una baja asistencia a centros preescolares, que con base en el censo del 2011 ubica en el $38.1 \%$ en niños de 4 años.

Destaca también que, de la oferta de centros preescolares en lo público equivale a un $86.1 \%$ y el resto son centros privados (p.77). Además, informa que las universidades públicas y privadas imparten carreras para estas áreas, aunque pocas están acreditadas. Con certeza se puede afirmar que al existir más universidades que ofrecen carreras vinculadas con educación, a aumentado el número de graduados en estas áreas, quienes probablemente, han encontrado 
mayores dificultades para trabajar Lo que sí hay es una amplia oferta docente, pero una marcada reducción de disponibilidad de plazas en el MEP. El documento reconoce que en la agenda política de los gobiernos costarricenses, la educación ocupa un lugar prioritario, pero los esfuerzos han sido insuficientes. Así en la educación preescolar en el nivel interactivo II, del ciclo materno infantil, la cobertura apenas es del $57.7 \%$, siendo que "el caso de niños y niñas con edades entre los 2 meses y los 4 años y 6 meses, los retos son mayores" (p.78). Cabe mencionar que el documento en cuestión establece que la primera infancia va de 0 a 8 años y la educación formal en Costa Rica se organiza considerando la etapa preescolar de 0 a 6 años, dentro de esta etapa está el ciclo Interactivo II que va de 4 años y 3 meses a 6 años y 3 meses.

Como se puede inferir, la obligatoriedad y universalización en el nivel preescolar no se cumple porque no hay una política nacional en educación que garantice este derecho con calidad y para toda la población. Los sectores más pobres urbanos y rurales se ven limitados a acceder a este derecho para la primera infancia $y$, quienes pueden acceder, no necesariamente se le garantiza que sea totalmente gratuita y de calidad. En sectores donde la educación pública no provee estos beneficios pueden suplirlos entidades privadas, pero éstas no son accesibles a todos, por los costos económicos para las familias.

El Informe considera que es necesario revisar y renovar programas, equiparar oportunidades y eliminar desigualdades, "se requiere una actualización de la práctica docente en todas las áreas del desarrollo infantil” (p.79), aunque reconoce que el país está en capacidad de avanzar en la educación preescolar a corto plazo. Ya en la administración Chinchilla Miranda se había integrado la Red Nacional de Cuido (REDCUDI), que incluye un programa que ya tiene años de funcionar como lo son los CEN - CINAI, los Hogares Comunitarios y los Centros de atención Integral. Esta Red evidencia que la primera etapa de la infancia no es 
atendida por el MEP, y surge precisamente porque es manifiesta la pobreza de hogares que tienen niños y niñas en estas edades y que tienen como cabeza de familia a mujeres". En síntesis, el $60 \%$ de los niños y niñas menores de 6 años (tres de cada cinco) forman parte de hogares pobres o vulnerables" (Cuarto Informe Estado de la Nación, 2013, p.86).

El régimen municipal contempla la creación de los Centros de Cuido y Desarrollo Infantil (CECUDI) en coordinación con el IMAS, pero en este caso el problema se presenta con la eficacia y las posibilidades reales de implementar estas iniciativas por parte de los gobiernos locales, que suelen tener limitaciones presupuestarias y de infraestructura, además de luchas políticas internas que apoyan o rechazan estos programas de acuerdo con quien esté al frente del gobierno central. Ante este panorama, han emergido centros maternales y guarderías privadas que no siempre cuentan con la idoneidad del caso.

El Informe pone en evidencia estas situaciones y demuestra que aunque normativamente se establezca la cobertura universal, obligatoria y financiada por el Estado, en la práctica desde estas primeras etapas de la infancia hay exclusión. También, evidencia que no hay continuidad entre los ciclos en el sistema educativo, porque hay niños y niñas que no tienen acceso a la educación preescolar, y si la tienen, no siempre es de calidad y en las mejores condiciones, lo que es muy lamentable porque "la edad preescolar es una etapa clave para sentar las bases que le permitirán a estos niños y niñas mayores grados de éxito personal y académico a lo largo de sus vidas" (Cuarto Informe del Estado de la Nación, 2013, p.90), considera el cuarto Informe sobre Educación.

\section{El derecho a la educación general básica y diversificada en Costa Rica}

La obligatoriedad y gratuidad de la educación primaria tiene larga data en nuestro país y se amplió con una reforma constitucional en el 2011 hasta el ciclo 
diversificado, obligando al Estado a destinar el 8\% de Producto Interno Bruto (PIB) con este propósito, colocando a Costa Rica entre los pocos países del área con una directriz en ese sentido. Nuevamente se muestra un desfase entre lo que se establece y lo que realmente se hace, ya que el cuarto Informe sobre Educación del 2013 dice que solo un 35\% de los estudiantes completó la educación secundaria (p.135), lo cual demuestra un aumento de la desigualdad.

En el capítulo 3, que es el que dedica a este tema empieza señalando algo interesante, el declive de la educación primaria y el aumento de la educación secundaria (p.136), como consecuencia de un descenso en la tasa de natalidad y un incremento de la población adolescente. Pero el MEP no ha dado suficiente cobertura para que los jóvenes concluyan con éxito el ciclo diversificado.

Los datos están en el Informe pero las interpretaciones que se hacen dependen de lo que se pretende defender o denunciar. Al respecto, el exministro de Educación anterior, el Dr. Leonardo Garnier (quien estuvo 8 años al frente de esa cartera ministerial) escribió un artículo de opinión en el Diario La Nación bajo el título de "Una inversión educativa que rinde frutos" (16 nov, 2014, p.30). En este artículo, Garnier comenta que hacia finales de los años 70 e inicios de los años 80 hubo una debacle educativa en secundaria donde la cobertura pasó de $60 \%$ a $55 \%$ y al final del siglo era de $59 \%$, pues mucha de las personas que estaban en edad colegial no ingresaron o dejaron inconclusa su secundaria. Pero, defiende que esos números han cambiado con los costarricenses que están en edad promedio de 15 años porque "la tasa de escolaridad bruta es hoy de $90.5 \%$ ", por lo que, según el exministro, si ha habido avances importantes para "que empiecen a desaparecer las brechas de desigualdad que han caracterizado nuestros mercados laborales en las últimas décadas". Describe también que la brecha de escolaridad entre estudiantes urbanos y rurales se redujo a un 7\%, así como la brecha de escolaridad entre estudiantes de hogares con mayores ingresos y la de 
menores ingresos "se ha reducido a un $17 \%$ mucho menos de la mitad". Y las brechas que separan a las familias con mayor nivel educativo de las que tienen un bajo nivel "se ha reducido del $68 \%$ al $29 \%$, menos de la mitad", de ahí que concluya Garnier que la inversión educativa ha rendido frutos.

Un día después en el mismo Diario aparece un artículo periodístico de Andrés Solano con el título de "50.000 adolescentes ticos entre 15 y 17 años trabajan", tomando como base una publicación de la Revista Investigación en Juventudes del Consejo de la Persona Joven. Afirma este periodista que el principal empleador es la empresa privada, o la iniciativa laboral formando una pequeña empresa. Concluye el artículo que "Los expertos consideran que existe una relación estrecha entre acceso a la educación y las posibilidades de conseguir un empleo bien remunerado. Según Solano quien remite a una encuesta realizada por la Revista en la que fundamenta su artículo, más de la mitad de las personas jóvenes entre 18 y los 24 años no han finalizado el bachillerato".

De acuerdo con el Cuarto Informe sobre la educación "sigue coexistiendo una baja cobertura del ciclo diversificado, que en el 2012 apenas llegó a un 47\%, la tasa de reprobación en secundaria mantiene su volatilidad y no muestra una tendencia clara hacia la mejora, mientras que los conocimientos y destrezas de la mayoría de los estudiantes se ubican en niveles de desempeño medios y bajos, tal como indican los resultados más recientes de las pruebas diagnósticas del MEP para noveno año y las pruebas PISA en el plano institucional" (Cuarto Informe del Estado de la Nación, 2013, p.137).

Aunque la información consignada antes de los artículos periodísticos y de opinión son del 2014 y los datos del Informe sobre la Educación son del 2013, la situación de la brecha social del país no se ha resuelto aún, por lo que, las dificultades para garantizar el derecho universal, obligatorio y gratuito de la educación, siguen 
presentes. Al respecto es pertinente considerar los datos que cita el periodista Ernesto Rivera, en el Semanario Universidad, con base en información del Instituto de Estadística y Censos (INEC) "para el 2014 muestran que, en 2014 los ingresos netos promedios de sector más rico fueron de 996.077 colones, mientras el ingreso neto promedio de los más pobres fue de 54.021 colones. Con estos números se revela que el quintil más pobre se reparte apenas el 3, $9 \%$ del ingreso nacional, mientras el quintil más favorecido se distribuye el $50.1 \%$ del ingreso disponible" (Rivera, 2014, p.4)

Le corresponde al Consejo Superior de Educación, órgano adscrito al MEP, la dirección general de la educación pública costarricense. En el 2008, este Consejo aprobó el documento El Centro Educativo de Calidad como Eje de la Educación Costarricense y cuyo propósito es impactar en la calidad educativa como clave para el desarrollo. Entre sus directrices, alude a una educación integral y de calidad como un derecho de todos, por lo que establece una política educativa universal, bajo el criterio de mayores niveles y exigencias educativas, desde la educación primaria hasta la secundaria con acceso a la universitaria, permitiendo que todos los sectores, en particular los más desfavorecidos tengan oportunidades de aprender durante toda la vida. En este sentido, el documento propone una educación abierta a las necesidades educativas especiales, étnicas, culturales, lingüísticas, religiosas, económicas, sociales. Esto es parte de lo que el Informe Delors llama "utopía necesaria", aunque en la práctica, a pesar del optimismo de personas como el exministro Garnier, las brechas sociales parecen estarse ensanchando, pues así lo muestran diferentes indicadores del desarrollo de país.

En años recientes ha habido una apertura de colegios públicos por todo el territorio, aunque existe una brecha entre los mismos centros de educación pública (académicos, técnicos, bilingües) y entre estos y la educación privada. Es pertinente aclarar que no se asume que la educación privada per se sea mejor que la pública, pues en la oferta educativa hay diferentes niveles de calidad. $\mathrm{Ni}$ 
que la calidad educativa de los colegios académicos es más baja que la de los otros tipos de colegios, pero si se evidencian diferencias.

No es el propósito del artículo detenerse en el análisis específico de estas diferencias, aunque amerita alguna reflexión al respecto. Tómese como ejemplo la educación técnica que viene teniendo una mayor cobertura y una gran demanda de parte del sector estudiantil que aspira a formar parte de un demandante mercado laboral amplio. Así se refleja en la siguiente cita del Cuarto Informe del Estado sobre la Educación "por condición de actividad, y según la Encuesta Nacional de Hogares (ENAHO) en 2012, más del $60 \%$ de la población con educación técnica completa estaba ocupado, un $4.6 \%$ estaba desempleado y un $29.6 \%$ se encontraba inactivo...Las personas ocupadas con educación técnica completa se emplean principalmente en el sector terciario de la economía, en actividades de comercio y servicios, con más del $60 \%$, le siguen el sector secundario, con $33.8 \%$ y el primario 5.7\%" (Cuarto Informe del Estado de la Nación, 2013, p.173).

Si se mira esta situación frente a las dificultades de conseguir trabajo en la actualidad, con educación secundaria completa o aún con la educación universitaria completa, los graduados de los colegios técnicos tienen mayores posibilidades laborales y salariales. Incluso hoy existen convenios entre el Ministerio de Educación Pública (MEP), el Instituto Nacional de Aprendizaje (INA) y Coalición de Iniciativas de Desarrollo (CINDE), para desarrollar programas conjuntos que interesan al sector empresarial.

Por otra parte, las diferencias entre la educación secundaria académica y técnica se muestra en la infraestructura, asesorías y eficiencia administrativa. Hoy una gran mayoría de los colegios técnicos cuentan con excelentes plantas físicas, con todo lo que se requiere para el aprendizaje de sus disciplinas, tales como 
laboratorios de cómputo, de idiomas, acceso a internet, mobiliario en buen estado, recursos tecnológicos, grupos de aprendizaje más pequeños. Esto se verifica por lo expresado por la MSc. Maricel Cox, Asesora Nacional de inglés, en el Departamento de Educación Técnica del MEP, informó que los asesores nacionales de la educación técnica recorren todo el país, facilitando capacitaciones a los docentes, con el apoyo directo de la empresa privada y Centros de Educación Superior como el Instituto Tecnológico y la Universidad Técnica Nacional (Entrevista personal, 13 febrero, 2015)

Cuando el Cuarto Informe alude a que "la posibilidad de promover ambientes de aprendizaje atractivos se ve entorpecida por graves problemas de infraestructura, entre los que sobresale la falta de condiciones mínimas, como servicios sanitarios con puertas, papel y agua" (Cuarto Informe del Estado de la Nación, 2013, p.136). Si bien no especifica si es un centro educativo académico, técnico, científico o bilingüe, por lo dicho antes, no se refiere a los colegios técnicos, sino una indirecta hacia los centros educativos académicos. En efecto, hay un abandono de la infraestructura en colegios académicos, no reciben la misma capacitación y asesoramiento, no cuentan con el apoyo directo de la empresa privada e incluso, los problemas administrativos y burocráticos son más evidentes. Súmese a lo dicho, que los docentes de especialidades técnicas, incluyendo los de idiomas, tienen mejores ventajas salariales, pues se les paga por lección hora y no por lección de 40 minutos como se hace en los académicos.

A propósito del área docente y administrativa en general, aunque cuenta con mejores incentivos salariales, sin embargo, sigue teniendo problemas administrativos en el reclutamiento del personal, lo que se manifiesta sobre todo al inicio de los ciclos lectivos, perjudicando la calidad de la educación. Considérese también algo que ya se acotó antes, la preparación de docentes procedentes de 
centros universitarios que no han certificado sus carreras, lo que también atenta contra la calidad de la educación.

El documento del Consejo Superior de Educación propone que la calidad de la educación debe ser apoyada por una gestión ágil, eficiente y amable, porque el objetivo es el aprendizaje y desarrollo personal y colectivo de los educandos. Sin embargo, el cuarto Informe sobre Educación evidencia "un sistema burocrático y prescriptivo, en el cual el exceso de estructuras, procedimientos y regulaciones resta autonomía y creatividad a directores y docentes para atender las necesidades de los estudiantes. En los centros persisten culturas organizativas poco favorables a la calidad y severas carencias en el uso de la información y de instrumentos de seguimiento y evaluación" (p.90).

Es importante que el informe mencione que en la parte de relaciones sociales entre los estudiantes se dan malos tratos, porque refleja situaciones psicológicas y sociológicas conflictivas en amplios sectores de la población estudiantil, que atentan contra un ambiente idóneo para que se vivencie un verdadero derecho a la educación, pero no se analizarán acá, aunque no se puede omitir los datos de una encuesta del 2011 a la que remite el documento que "reveló que más del $60 \%$ de los alumnos reporta malos tratos (insultos, malas palabras, descalificaciones 0 gritos) entre compañeros" (Cuarto Informe del Estado de la Nación, 2013, p.137). Por último hay otras importantes manifestaciones que demuestran que el derecho a la educación en el ámbito intercultural no se cumple como debería de ser y, que, por el contrario, se están ensanchando las brechas sociales, específicamente al que mencionar a los grupos indígenas, que también tienen marcadas diferencias entre sí, pues algunas etnias se encuentran más excluidas que otras, pero este es un amplio campo, que amerita mayor atención y que excede los intereses de este artículo. 


\section{Conclusión}

La garantía del derecho a la educación implica un tema muy complejo y delicado, no debe ser abordado solo por la política, las normas jurídicas o de Declaraciones y manifiestos. Quienes hablan de este derecho son personas que ya han pasado por un proceso escolar y que pretenden establecer directrices en la educación formal para transmitirlas a quienes se pretende escolarizar. Irónicamente a los principales actores de ese proceso, empezando por la niñez y adolescencia, no se les da el protagonismo que merecen por cuanto se les participa parcialmente o se les niega totalmente su papel como generaciones presentes y futuras.

Es inevitable que el derecho a la educación se encuentre con diversos obstáculos, porque la educación, particularmente la escolarizada, por su misma naturaleza, forma parte de los medios más frecuentes que utilizan la autoridad y la tradición cultural para mantener su hegemonía. Sin olvidar que cuando se inicia la escolarización se inicia la transición de niños y niñas hacia la vida pública, aunque está claro que ésta no es la única fuente de educación, la introducción es gradual y estará mediada por figuras de autoridad y aunque las teorías pedagógicas modernas insisten en que la educación debe estar al servicio del educando (sea niño, joven o adulto), parece ser que, con alguna frecuencia se olvidan sus necesidades y sus derechos.

Cuando se habla de derechos humanos en el ámbito Estatal, siempre está presente la sombra de lo económico, donde a quienes les corresponde ejercer los mecanismos del poder les cuesta hacer el balance entre crecimiento y equidad. Porque constantemente se habla ahora de crecimiento sostenible, pero la desigualdad también crece, lo que atenta contra la dignidad de las personas, en particular las más desfavorecidas, así un derecho medular como lo es el de la educación lamentablemente no es inclusivo en amplias regiones del mundo y 
nuestro país no es la excepción, a pesar de los valiosos esfuerzos que se han realizado.

Los datos comentados demuestran esa exclusión, demuestran escolarización obligatoria inconclusa, gratuidad cuestionada, pues el costo de la vida pasa la factura a las familias pobres al inicio y durante el ciclo lectivo. La conclusión de la educación diversificada no garantiza el acceso a la educación universitaria, ni al mundo laboral. Salvo el caso comentado de los egresados de los colegios técnicos, provenientes de hogares con ingresos bajos y medios, que se han visto beneficiados con este tipo de sistema educativo, que abre posibilidades reales de iniciativas emprendedoras propias que garantizan una mejor calidad de vida. Sin embargo, no deja de ser preocupante la enorme injerencia en los planes de estudio y especialidades técnicas a desarrollar, que tiene el sector empresarial, porque puede ser este el camino para una privatización y mercantilización de la educación.

El derecho a la educación no debe mercantilizarse, debe ser integral, inclusiva y dignificante, éste aún es parte de esa utopía necesaria, que no debemos de abandonar, sino más bien canalizarla hacia una realidad en un futuro cercano. 


\section{Referencias}

Arendt, H. (1996). Entre el pasado y el futuro. Barcelona: Península.

Consejo Superior de Educación. (2008). El Centro Educativo de Calidad como Eje de la Educación.

Costa Rica. (1949). Constitución Política de la República de Costa Rica. San José. Recuperado de www.constitution.org/cons/costaric.htm

Costa Rica. (1957). Ley Fundamental de Educación. San José. Recuperado de www.apse.or.cr/webapse/legdoc/leg02htm

Cox, M. (13 de febrero de 2015). Entrevista realizada Asesora Nacional de inglés, Departamento de Educación Técnica, Ministerio de Educación Pública.

USA. (1948). Declaración Universal de Derechos Humanos. New York: ONU. Recuperado de www.un.org/es7document/udhr/

Delors, J. (1999). La educación encierra un tesoro. Paris - Madrid: UNESCO, Santillana.

Garnier, L. (16 de noviembre 2014). "Una inversión educativa que rinde frutos”. En La Nación, Costa Rica, p.30 A.

Méndez, M.V. (2014). El derecho a la educación y las edades mínimas de protección legal en Centroamérica. Costa Rica: sin datos de edición.

Muñoz, V. (2009). El mar entre la niebla: el camino de la educación hacia los derechos humanos. Costa Rica: Luna hibrida ediciones.

Programa Estado de la Nación. (2013). Cuarto Informe Estado de la Educación. San José, Programa Estado de la Nación.

Rivera, E. (12 al 18 noviembre del 2014). "La pobreza tiene remedio". Semanario Universidad, p.5.

Solano, A. (2014). “50.000 adolescentes ticos entre 15 y 17 años trabajan". En La Nación, Costa Rica, p.8A.

Torres, R.M. (2005). 12 tesis para el cambio educativo. Movimiento Fe y Alegría. 
El derecho a la educación en Costa Rica: entre la realidad y la utopía

Carlos Alberto Rodríguez Ramírez

UNESCO. (2011). ¿Qué es la UNESCO? Recuperado de www.unescogetafe.org/index.php/sobre-unesco-getafe/que-es-la-unesco 